\title{
Effect of AGO2 Depletion on Cell Migration of A172 Brain Cancer Cell
}

\author{
Sujin Kim ${ }^{1}$, Yoonseo Kim², Wonse Kim³ ${ }^{3}$ Woo Rin Lee ${ }^{4, *}$ \\ ${ }^{1}$ Loomis Chaffee School, Connecticut, United States \\ ${ }^{2}$ Saint Stephen's Episcopal School, Texas, United States \\ ${ }^{3}$ Saint Paul's School, New Hampshire, United States \\ ${ }^{4}$ Department Biological Science, University of Suwon, Hwaseong, Republic of Korea
}

Email address:

sujink748@gmail.com (S. Kim), kimyoonseo509@gmail.com (Y. Kim), wonsekim1017@gmail.com (W. Kim), gosyber1@gmail.com (W. R. Lee)

${ }^{*}$ Corresponding author

\section{To cite this article:}

Sujin Kim, Yoonseo Kim, Wonse Kim, Woo Rin Lee. Effect of AGO2 Depletion on Cell Migration of A172 Brain Cancer Cell. Cancer Research Journal. Vol. 8, No. 3, 2020, pp. 51-56. doi: 10.11648/j.crj.20200803.12

Received: September 7, 2020; Accepted: September 29, 2020; Published: October 16, 2020

\begin{abstract}
AGO2, a protein-coding and miRNA regulating gene, has recently been identified as a phenomenal biomarker in cancer development research. Its role varies in different cancers, acting as either a tumor suppressor or an oncogene. However, no research has been conducted on the correlation between $\mathrm{AGO} 2$ and brain cancer yet. The aim of this study was to define the association of AGO2 amplification and brain cancer patient's survival and to investigate the functional role of AGO2 in brain cancer cell migration. 20 brain cancer studies with a total of 6164 patient samples via cBioPortal were analyzed. After the patients were divided into two groups: $\mathrm{AGO} 2$ amplified group and $\mathrm{AGO} 2$ non-amplified group, the overall patients' survival rate was analyzed by Kaplan-Meier. The positive correlation between the AGO2 amplified group and survival rates was discovered. To find out how AGO2 amplification increased patients' survival rates, we performed an in vitro assay to silence AGO2 expression using two siRNAs targeting AGO2 in A172 brain cancer cell line. The wound-healing assay was performed to show that both siRNAs targeting AGO2 promoted cell migration of A172. Therefore, AGO2 may function as a tumor suppressor and enhances survival rates of brain cancer patients by inhibiting cancer cell migration. Further investigation of the mechanisms affecting $\mathrm{AGO} 2$ dysregulation will provide insights into the molecular differences underpinning brain cancer patient's survival rate.
\end{abstract}

Keywords: AGO2, Brain Cancer, siRNA, RT-PCR, Cell Migration

\section{Introduction}

Brain cancer is the fifth leading cause of cancer-related deaths among men aged 40 59 years in the world [1]. Over 700,000 Americans are living with a brain tumor, of which $30.2 \%$ are malignant [2]. The average survival rate for all malignant brain tumor patients is only $36 \%$ [3].

Argonaute 2 (AGO2) is a protein coding gene located at $8 \mathrm{q} 24.3$ [4]. Its main function, like other argonaute proteins, is RNA-induced silencing complex (RISC), also known as RNA interference [5]. As part of the RISC, AGO2 binds with the miRNA or the siRNA and approaches the target mRNA [5]. AGO2 then binds the miRNA or the siRNA to its corresponding sequence in the target mRNA to inhibit the translation process [5]. The RISC complex eventually cleaves the targeted sections of the mRNA, successfully silencing the gene [5]. AGO2 also functions in many other processes such as angiogenesis, cell differentiation, and the regulation of the immune system [6-8].

AGO2 functions have been contradictory in different types of cancers as either tumor suppressor genes or oncogenes. In breast, lung, and gastric cancers, $\mathrm{AGO} 2$ functioned as a tumor suppressor gene that regulates miRNA to reduce its alterations, prohibiting cancer development $[9,10]$. On the other hand, AGO2 functioned as an oncogene in esophageal, prostate, cervical, and bladder cancers [11-13]. In cervical 
cancer alone, three different studies showed that AGO2 was an oncogene through promotion of TERT activity and malignancies in HeLa cells during AGO2 up-regulation [14]. A previous study determined a correlation between AGO2, TERT, and TERC in various cancers [15]. However, the role of AGO2 in brain cancer is poorly studied.

In this study, to elucidate the functional role of $\mathrm{AGO} 2$ in brain cancer, we demonstrate that amplification of $\mathrm{AGO} 2$ increased patient's survival rates. Depletion of AGO2 expression by siRNA activates cell migration in vitro in A172 brain cancer cell line.

\section{Materials and Methods}

\subsection{Patient Survival Analysis with cBioPortal}

The cBioPortal for cancer genomics is an open-access resource. (http://www.cbioportal.org). This database provides visualization and analyzing tools for more than 6,000 tumor samples from 290 cancer studies in TCGA pipeline [16]. The search interface enables researchers to analyze genetic alterations across samples from other cancer studies with specific genes [17]. The term "AGO2" was searched in cBioPortal database; a total of 6,164 samples from 20 brain cancer studies and a cross-cancer summary was obtained. The search parameters included alterations such as amplifications, deep deletions, and mutations. Overall survival (OS) was calculated on the basis of cBioPortal's online instruction.

\subsection{Cell Line and Culture}

Human brain cancer cell line A172 was obtained from the Korean Cell Line Bank. A172 cells were maintained in Gibco ${ }^{\circledR}$ RPMI-1640 medium (Life Technologies) supplemented with $10 \%$ HyClone fetal bovine serum (Thermo Fisher) and 1\% penicillin and streptomycin in a 5\% $\mathrm{CO}_{2}$ atmosphere at $37^{\circ} \mathrm{C}$. Cells were kept in culture for four or fewer passages.

\subsection{RNA Interference}

Predesigned two siRNAs (27161-1, 27161-2) were purchased from Bioneer to knockdown AGO2. The negative control scrambled siRNA med GC (12935-300) was purchased from Invitrogen. Cells were transfected with 20 $\mathrm{nM}$ siRNAs using lipofectamine RNAimax reagent (Invitrogen) with 1:3 ratio of siRNA $(\mu \mathrm{g})$ to lipofectamine $(\mu \mathrm{l})$.

\subsection{Total RNA Isolation and Reverse Transcription}

Total cellular RNA was extracted with Total RNA extraction spin kit (Intron) and treated with DNase I (Invitrogen). The cDNA was reverse-transcribed from $1 \mu \mathrm{g}$ of total RNA using oligo (dT) primers according to the manufacturer's protocol (Enzynomics).

\subsection{Polymerase Chain Reaction}

Polymerase chain reaction was performed in $20 \mu \mathrm{l}$ reaction with PCR-Premix (Bioneer). The PCR mixture was prepared according to the manufacturer's protocol. The primers from the previous study were used for the amplification of cDNA from each of two human genes [18]. PCR was performed with cycles of $94^{\circ} \mathrm{C}$ for $15 \mathrm{~s}, 55^{\circ} \mathrm{C}$ to $60^{\circ} \mathrm{C}$ for $15 \mathrm{~s}$, and $74^{\circ} \mathrm{C}$ for $30 \mathrm{~s}$. The forward primer 5'GTTTGACGGCAGGAAGAATCT -3' and reverse primer 5'AGGACACCCACTTGATGGACA -3' yielded a 131-base pair (bp) product from $\mathrm{AGO} 2$ using an annealing temperature $55^{\circ} \mathrm{C}$. For GAPDH, a (176) bp product was produced by forward primer 5'- TGGAGAAGGCTGGGGCTCAT -3' and reverse primer 5'- GACCTTGGCCAGGGGTGCTA -3'.

\subsection{Gel Electrophoresis and Data Acquisition}

The $1.2 \%$ agarose gels were prestained with RedSafe nucleic acid staining reagent (Intron). Aliquots taken from PCR reactions were electrophoresed on agarose gels at 100 volts for $25 \mathrm{~min}$. The gel electrophoresis images were captured with a digital camera, and the densitometry feature of Image studio ver 5.2 was used to measure band intensities. The relative intensities of $\mathrm{AGO} 2$ bands were normalized by the GAPDH band.

\subsection{Cell Migration Assay}

A sterile plastic micropipette tip was used to stimulate in vitro wound by creating a straight-edged, cell-free zone across the cell monolayer in the well. A gap width of $0.5 \mathrm{~mm}$ was observed at $\mathrm{x} 10$ magnification. After creating the scratch, the monolayer was washed with RPMI 1640 medium to remove cell debris. After RPMI 1640 media was added, the cells were incubated in $37^{\circ} \mathrm{C}, 5 \% \mathrm{CO}_{2}$ incubator (Eppendorf). Migration processes were documented by taking digital photographs of the gap using bright-field microscopy (Nikon) at indicated times. The wound area was calculated using ImageJ software. Each experimental condition was evaluated in a triplicate.

\subsection{Statistical Analysis}

All statistical analysis was performed using SPSS 13.0 (SPSS) and Excel (Microsoft). Quantitative data were expressed as mean \pm SD (standard deviation). Survival curves were plotted using the cBioPortal. All results are displayed with $P$ values from a log-rank test. For gene expression and migration analysis, Student's $t$-test was used to compare two samples. $P$ values of $<0.05$ were considered to be statistically significant.

\section{Result}

\subsection{Amplification of AGO2 Increases the Overall Survival of Brain Cancer Patients}

Gene amplification has been widely observed in human tumors [19]. This process acts as one of the oncogeneactivating genetic mechanisms and frequently shows an aggressive behavior of the tumor and poor prognosis [19, 
20]. By employing public clinical data from cBioPortal, the AGO2 gene on chromosome 8q24 was frequently amplified in brain cancer patient population. The AGO2 gene was amplified in $1.75 \%(91 / 5199)$ of patients with brain cancer (Figure 1). When patients were divided into two groups (AGO2 amplified group and AGO2 non-amplified group), AGO2 gene amplification showed a significant association with increased overall survival ( $p=4.37 \mathrm{e}-6$ ), suggesting that the amplified AGO2 may be a protective factor of brain cancer (Figure 1). The median survival month of amplified AGO2 showed 67.4 month compared to 23.3 months in nonamplified AGO2 group (Figure 1). This implies that AGO2 amplification may correlate with inhibiting brain cancer progression.

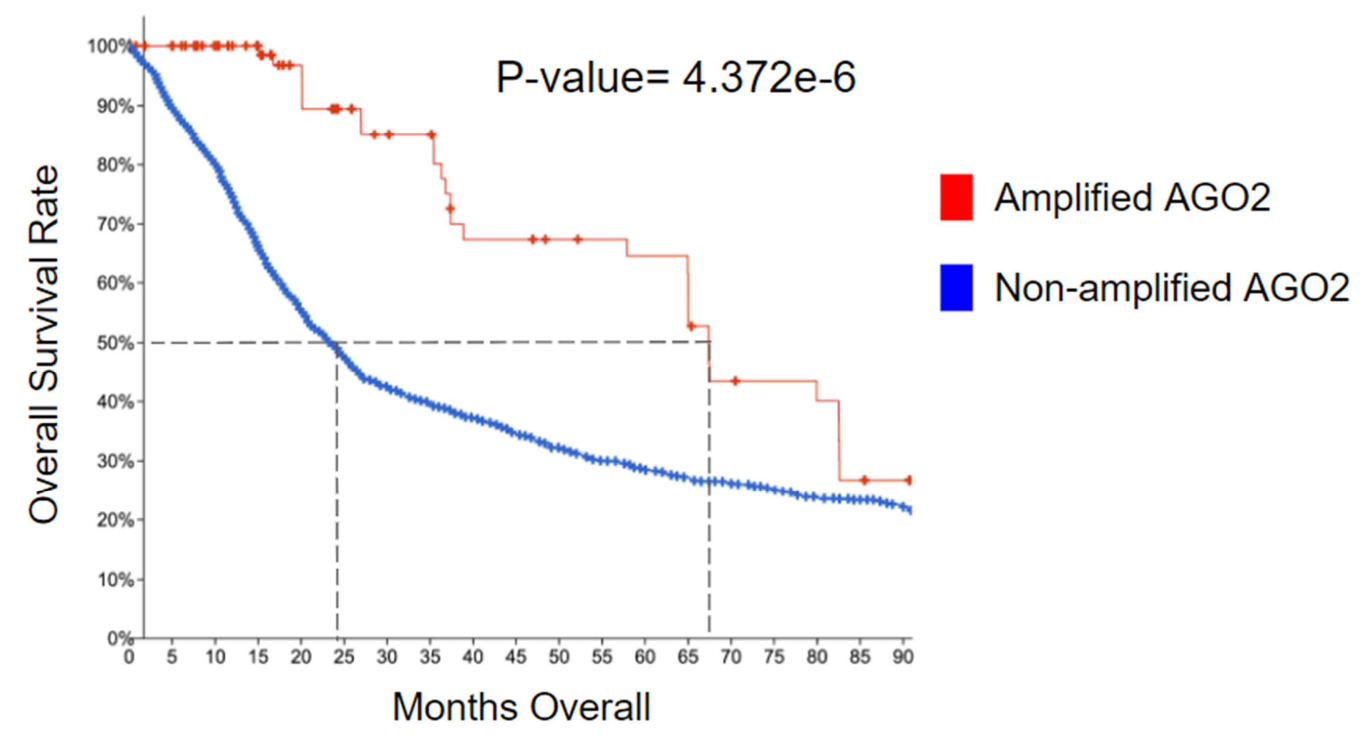

Figure 1. AGO2 amplification enhances patient survival rate. The AGO2 amplified group (red) and wild type group (blue) were 91 and 5108 patients respectively. Out of the total patients, the amplified group lived a median 67.4 months overall. The wild type group lived a median 23.3 months.

\section{2. siRNA Targeting AGO2 Decreased mRNA Expression Level of $A G O 2$}

Gene amplification is associated with increased expression of their respective mRNA and protein [21]. Previous study showed that changes in $\mathrm{AGO} 2$ gene copy can affect expression levels and correlate with high-risk disease in multiple myeloma [22]. To understand the functional role of AGO2 in cancer, A172 brain cancer cells were transfected with two different small interfering RNAs (siRNAs) against AGO2. Off-target effects can be mediated by the sequence-

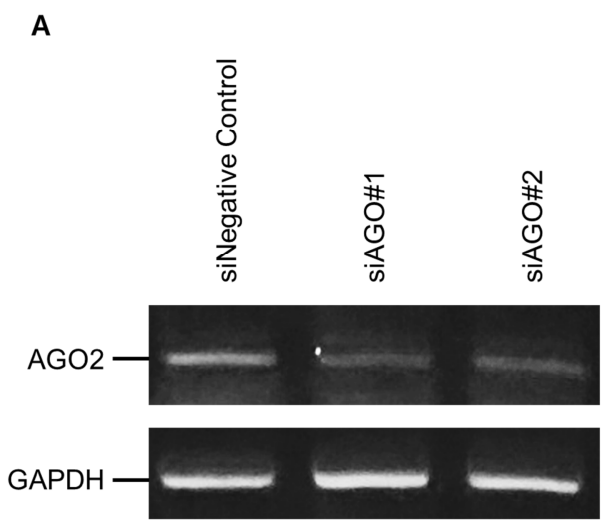

specific interaction between the siRNA seed regions and complementary sequence of the target [23]. Therefore, one siRNA for negative control and two different siRNAs for targeting $\mathrm{AGO} 2$ were used in this experiment to validate the effect of siRNA on AGO2 expression level. The effect of siRNA transfections on AGO2 expression was confirmed using quantitative polymerase chain reaction (qPCR) (Figure 2). The result indicates that two different siRNA targeting AGO2 decreased mRNA expression level of AGO2 in A172 cells.

B

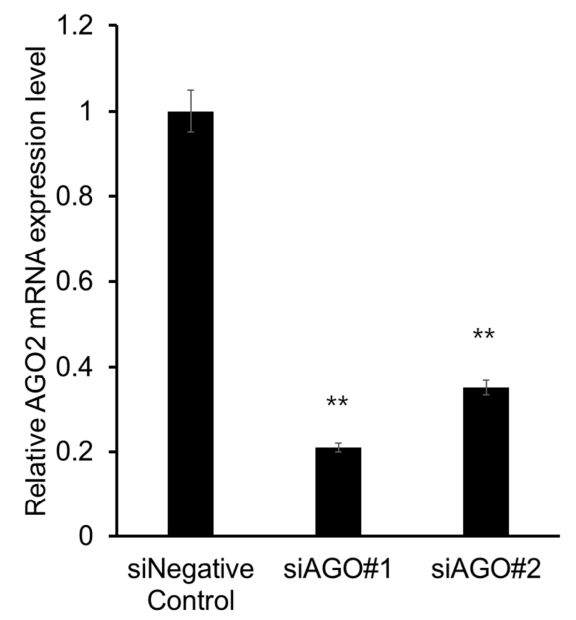

Figure 2. siRNA targeting AGO2 decreased the mRNA of AGO2 of A172 cells. (A) qPCR analysis showing the mRNA levels of siNegative control, siAGO\#1, and siAGO\#2 transfected A172 cells at $48 \mathrm{~h}$ after transfection. (B) qPCR analysis of AGO2 mRNA levels at $48 \mathrm{~h}$ after transfection $(n=3$, mean $\pm S D)$. Student's t test, ${ }^{* *}<$ p 0.01 . 


\subsection{AGO2 Depletion Promoted Migration in A172 Cells}

The tumor metastasis has been detected in the late stage of the cancer, mostly leading to death [24]. Cancer cell migration supports tumor metastasis: tumor cell migration begins when a tumor cell leaves the primary site, and arrives at and survives in distant tissues and organs [25]. In addition, a previous research has found a stronger association of predicted migration levels with the patient's survival compared to the predicted proliferation levels in transcriptomic analysis [26]. Since this previous study indicated that $\mathrm{AGO} 2$ regulates cancer cell migration in prostate and breast cancer, we decided to evaluate the functional role of AGO2 on brain cancer cell migration [27, 28]. The photograph of migrated cells was captured at $0 \mathrm{~h}, 6$ $\mathrm{h}, 30 \mathrm{~h}$ after a "wound gap" in a cell monolayer was created by scratching (Figure 3a). The result indicates that the percentage of wound closure was significantly increased in siAGO2 transfected cells at $30 \mathrm{~h}$ (Figure $3 \mathrm{~b}$ ). Since AGO2 depletion by siAGO\#1 and siAGO\#2 promoted cell migration of A172 cells, this result indicates that AGO2 elicits its inhibitory effect on migration in brain cancer.
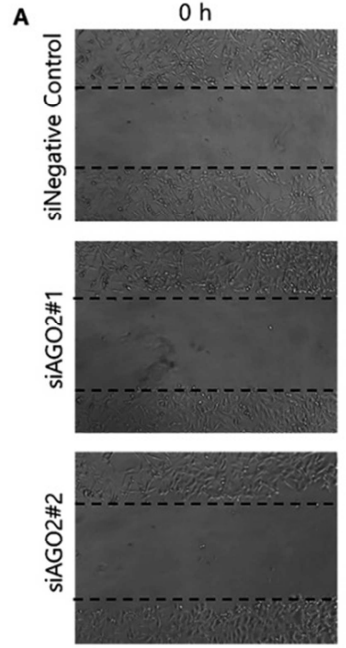

$6 \mathrm{~h}$
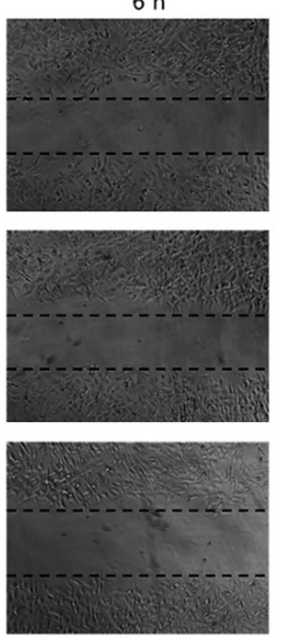

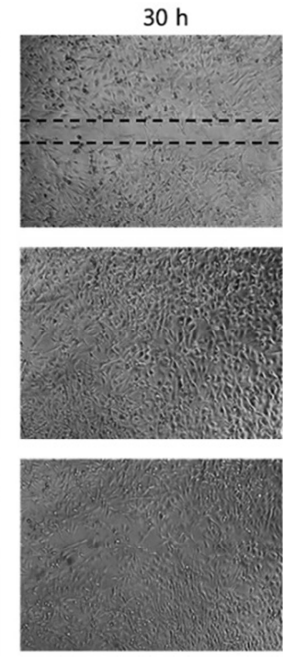

B

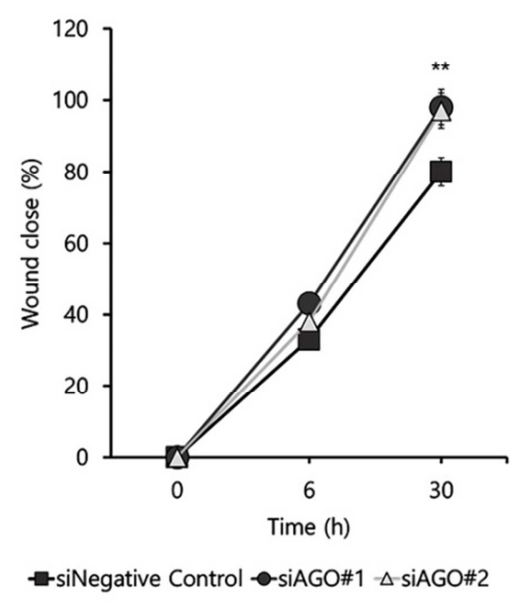

Figure 3. AGO2 depletion by siRNA transfection promoted the cell migration of A172 cells. (A) Cells were scraped with a pipette tip and transfected with siNegative control, siAGO\#1, and siAGO\#2 for $30 \mathrm{~h}$. Cells were imaged under a light microscope (magnification of x10). (B) Cell migration was quantified by measuring wound closure areas. Quantitative data are presented $(n=3$, mean $\pm S D)$. Student's t test, ${ }^{*}<p 0.01$.

\section{Discussion}

8q, a chromosome in which $\mathrm{AGO} 2$ is located, amplification of this position was observed to increase aggressive tumor phenotypes, developing into advanced metastatic and hormone refractory tumors, and diminish patient survival rates in various cancers such as prostate and kidney cancers [29, 30]. Via cBioPortal, copy number variation of genes located near $\mathrm{AGO} 2$ in the 8q chromosome, such as MYC, RECQL4, RAD21, NDRG1, and RSPO2, was seen to positively affect patient survival rates in brain cancer (data not shown). Therefore, the effect of amplification of these genes on brain cancer patients' survival should be further investigated.

Specific gene expression silencing by RNAi is a mechanism of transcriptional regulation in the cells. This can be mediated by small RNA with 21-23 nucleotides length called siRNA. When we depleted AGO2 expression level with two siRNAs targeting AGO2, migration of A172 cells was enhanced. However, the effects of $\mathrm{AGO} 2$ expression on cell migration and metastasis have varied greatly between several cancer studies. On one hand, studies have found that AGO2 encourages metastasis in prostate, liver, and hypopharyngeal cancer [31-33]. A study in prostate cancer found that down regulation of AGO2 suppressed metastasis [31]. A recent study in liver cancer revealed that $\mathrm{AGO} 2$ binds to FAK promoter and expedites tumor metastasis [32]. A study in hypopharyngeal cancer found that $\mathrm{AGO} 2$ acts as an oncogene that promotes both tumorigenesis and cell migration by regulating FAK/PI3K/AKT signaling pathway [33].

On the other hand, AGO2 was observed to inhibit migration behavior in melanomas and breast cancer [9, 34]. A study in melanoma found that $\mathrm{AGO} 2$ re-expression reduces the migration behavior significantly by $\sim 20 \%$ [34]. In breast cancer, the AGO protein was observed to repress miRNA translation [9]. However, conflicting results were reported in Hepatocellular Carcinoma (HCC). One study found that $\mathrm{AGO} 2$ promotes tumor metastasis through up-regulating focal adhesion kinase expression while another showed that regulating $\mathrm{AGO} 2$ via miR-184 promotes proliferation and migration $[32,35]$.

Cell adhesion proteins and migration behavior are closely related [36]. MCF-7-Ago2-WT cells have a "scattered" phenotype reminiscent of the epithelial-to-mesenchymal transition process noted previously [37]. This includes reduced cell-cell adhesion, lower levels of the adhesion molecules E-cadherin and $\beta$-catenin, and a greater degree of 
migratory capabilities as measured by the wound healing assay [37]. Through the AGO2-immunoprecipitation assay, it was discovered that the adhesion molecules, E-Cadherin and EpCAM, were upregulated by miR-96 in the prostate cancer bone metastasis samples [38]. This gives the tumor an advantage during the late stages of metastasis in the Mesenchymal and Epithelial Transition (MET) [38]. Future work should explore the correlation of these adhesion molecules and AGO2 expression level in brain cancer.

\section{Conclusion}

This study has several acknowledged limitations, the first being that associations between the AGO2 gene and other genes or genetic mechanisms were not validated, and that the protein expression level was not analyzed. Also, further experiments to identify the genetic and epigenetic regulatory mechanisms of the $\mathrm{AGO} 2$ gene are needed. Another limitation was the sample size of the AGO2 gene amplification group $(n=91)$, which was small for reasonable statistical inferences. In addition, the effect of $\mathrm{AGO} 2$ depletion on brain cancer cells should be validated by different brain cancer cell lines to evaluate both its prognostic significance and to explore the possibility of modulating this gene expression for therapeutic purposes. Despite these drawbacks, we demonstrated the prognostic potential of the AGO2 gene in brain cancer with cBioPortal data. When the AGO2 gene was depleted by siRNA, it promoted cancer cell migration. Overall, AGO2 may function as a tumor suppressor and enhances the survival rates of brain cancer patients.

\section{References}

[1] Barnholtz-Sloan JS, Ostrom QT, Cote D. Epidemiology of brain tumors. Neurol Clin. 2018; 36: 395-419.

[2] Miranda-Filho A, Piñeros M, Soerjomataram I, Deltour I, Bray F. Cancers of the brain and CNS: global patterns and trends in incidence. Neuro Oncol. 2017; 19: 270-280.

[3] Bondy ML, Scheurer ME, Malmer B, Barnholtz-Sloan JS, Davis FG, Il'yasova $\mathrm{D}$, et al. Brain tumor epidemiology: consensus from the Brain Tumor Epidemiology Consortium. Cancer. 2008; 113: 1953-1968. doi: 10.1002/cncr.23741

[4] Höck J, Meister G. The Argonaute protein family. Genome Biol. 2008; 9: 210.

[5] Rand TA, Petersen S, Du F, Wang X. Argonaute2 cleaves the anti-guide strand of siRNA during RISC activation. Cell. 2005; 123: 621-629.

[6] Shekar PC, Naim A, Sarathi DP, Kumar S. Argonaute-2-null embryonic stem cells are retarded in self-renewal and differentiation. J Biosci. 2011; 36: 649-657.

[7] Wu S, Yu W, Qu X, Wang R, Xu J, Zhang Q, et al. Argonaute 2 promotes myeloma angiogenesis via microRNA dysregulation. J Hematol Oncol. 2014; 7: 40.

[8] Zhang X, Zhao H, Gao S, Wang W-C, Katiyar-Agarwal S,
Huang H-D, et al. Arabidopsis Argonaute 2 regulates innate immunity via miRNA393 (*)-mediated silencing of a Golgilocalized SNARE gene, MEMB12. Mol Cell. 2011; 42: 356366.

[9] Bellissimo T, Tito C, Ganci F, Sacconi A, Masciarelli S, Di Martino G, et al. Argonaute 2 drives miR-145-5p-dependent gene expression program in breast cancer cells. Cell Death Dis. 2019; 10: 17.

[10] Zhang J, Fan X, Wang C, Liu B, Li Q, Zhou X. Up-regulation of Ago2 expression in gastric carcinoma. Med Oncol. 2013; 30: 628 .

[11] Yoo NJ, Hur SY, Kim MS, Lee JY, Lee SH. Immunohistochemical analysis of RNA-induced silencing complex-related proteins AGO2 and TNRC6A in prostate and esophageal cancers. APMIS. 2010; 118: 271-276.

[12] Guo J, Lv J, Liu M, Tang H. miR-346 Up-regulates Argonaute 2 (AGO2) Protein Expression to Augment the Activity of Other MicroRNAs (miRNAs) and Contributes to Cervical Cancer Cell Malignancy. J Biol Chem. 2015; 290: 30342 30350 .

[13] Yang F-Q, Huang J-H, Liu M, Yang F-P, Li W, Wang G-C, et al. Argonaute 2 is up-regulated in tissues of urothelial carcinoma of bladder. Int J Clin Exp Pathol. 2014; 7: 340-347.

[14] Laudadio I, Orso F, Azzalin G, Calabrò C, Berardinelli F, Coluzzi E, et al. AGO2 promotes telomerase activity and interaction between the telomerase components TERT and TERC. EMBO Rep. 2019; 20.

[15] Ye Z, Jin H, Qian Q. Argonaute 2: A novel rising star in cancer research. J Cancer. 2015; 6: 877-882.

[16] Gao J, Aksoy BA, Dogrusoz U, Dresdner G, Gross B, Sumer $\mathrm{SO}$, et al. Integrative analysis of complex cancer genomics and clinical profiles using the cBioPortal. Sci Signal. 2013; 6: pl1.

[17] Cerami E, Gao J, Dogrusoz U, Gross BE, Sumer SO, Aksoy $\mathrm{BA}$, et al. The cBio cancer genomics portal: an open platform for exploring multidimensional cancer genomics data. Cancer Discov. 2012; 2: 401-404.

[18] Wang S, Sun X, Yi C, Zhang D, Lin X, Sun X, et al. AGO2 Negatively Regulates Type I Interferon Signaling Pathway by Competition Binding IRF3 with CBP/p300. Front Cell Infect Microbiol. 2017; 7: 195.

[19] Matsui A, Ihara T, Suda H, Mikami H, Semba K. Gene amplification: mechanisms and involvement in cancer. Biomol Concepts. 2013; 4: 567-582.

[20] Lockwood WW, Chari R, Coe BP, Girard L, Macaulay C, Lam $\mathrm{S}$, et al. DNA amplification is a ubiquitous mechanism of oncogene activation in lung and other cancers. Oncogene. 2008; 27: 4615-4624.

[21] Jia Y, Chen L, Jia Q, Dou X, Xu N, Liao DJ. The wellaccepted notion that gene amplification contributes to increased expression still remains, after all these years, a reasonable but unproven assumption. J Carcinog. 2016; 15: 3.

[22] Zhou Y, Chen L, Barlogie B, Stephens O, Wu X, Williams $\mathrm{DR}$, et al. High-risk myeloma is associated with global elevation of miRNAs and overexpression of EIF2C2/AGO2. Proc Natl Acad Sci USA. 2010; 107: 7904-7909. 
[23] Jackson AL, Burchard J, Schelter J, Chau BN, Cleary M, Lim $\mathrm{L}$, et al. Widespread siRNA "off-target" transcript silencing mediated by seed region sequence complementarity. RNA. 2006; 12: 1179-1187.

[24] Seyfried TN, Huysentruyt LC. On the origin of cancer metastasis. Crit Rev Oncog. 2013; 18: 43-73.

[25] Yang Y, Zheng H, Zhan Y, Fan S. An emerging tumor invasion mechanism about the collective cell migration. Am J Transl Res. 2019; 11: 5301-5312.

[26] Nair NU, Das A, Rogkoti V-M, Fokkelman M, Marcotte R, de Jong CG, et al. Migration rather than proliferation transcriptomic signatures are strongly associated with breast cancer patient survival. Sci Rep. 2019; 9: 10989.

[27] Kojima S, Enokida H, Yoshino H, Itesako T, Chiyomaru T, Kinoshita T, et al. The tumor-suppressive microRNA-143/145 cluster inhibits cell migration and invasion by targeting GOLM1 in prostate cancer. J Hum Genet. 2013; 59: 78-87.

[28] Götte M, Mohr C, Koo CY, Stock C, Vaske AK, Viola M, et al. miR-145-dependent targeting of junctional adhesion molecule A and modulation of fascin expression are associated with reduced breast cancer cell motility and invasiveness. Oncogene. 2010; 29: 6569-6580.

[29] El Gammal AT, Brüchmann M, Zustin J, Isbarn H, Hellwinkel OJC, Köllermann J, et al. Chromosome 8p deletions and 8q gains are associated with tumor progression and poor prognosis in prostate cancer. Clin Cancer Res. 2010; 16: 5664.

[30] Mehrazin R, Dulaimi E, Uzzo RG, Devarjan K, Pei J, Smaldone $\mathrm{MC}$, et al. The correlation between gain of chromosome $8 \mathrm{q}$ and survival in patients with clear and papillary renal cell carcinoma. Ther Adv Urol. 2018; 10: 3-10.
[31] Wang M, Ren D, Guo W, Wang Z, Huang S, Du H, et al. Loss of miR-100 enhances migration, invasion, epithelialmesenchymal transition and stemness properties in prostate cancer cells through targeting Argonaute 2. Int J Oncol. 2014; 45: $362-372$.

[32] Cheng N, Li Y, Han Z-G. Argonaute2 promotes tumor metastasis by way of up-regulating focal adhesion kinase expression in hepatocellular carcinoma. Hepatology. 2013; 57: 1906-1918.

[33] Zhang Y, Wang B, Chen X, Li W, Dong P. AGO2 involves the malignant phenotypes and FAK/PI3K/AKT signaling pathway in hypopharyngeal-derived FaDu cells. Oncotarget. 2017; 8: $54735-54746$.

[34] Völler D, Reinders J, Meister G, Bosserhoff AK. Strong reduction of $\mathrm{AGO} 2$ expression in melanoma and cellular consequences. Br J Cancer. 2013; 109: 3116-3124.

[35] Huang W, Huang F, Lei Z, Luo H. LncRNA SNHG11 Promotes Proliferation, Migration, Apoptosis, and Autophagy by Regulating hsa-miR-184/AGO2 in HCC. Onco Targets Ther. 2020; 13: 413-421.

[36] Huttenlocher A, Sandborg RR, Horwitz AF. Adhesion in cell migration. Curr Opin Cell Biol. 1995; 7: 697-706.

[37] Adams BD, Claffey KP, White BA. Argonaute-2 expression is regulated by epidermal growth factor receptor and mitogenactivated protein kinase signaling and correlates with a transformed phenotype in breast cancer cells. Endocrinology. 2009; 150: 14-23.

[38] Voss G, Haflidadóttir BS, Järemo H, Persson M, Catela Ivkovic T, Wikström P, et al. Regulation of cell-cell adhesion in prostate cancer cells by microRNA-96 through upregulation of E-Cadherin and EpCAM. Carcinogenesis. 2020; 41: 865874. 